\title{
Le rire cordial dans les demandes téléphoniques par de jeunes infirmières à l'hôpital
}

Cordial laughter in young nurses' telephone requests at the hospital

\section{Esther González-Martínez et Cécile Petitjean}

\section{(2) OpenEdition}

\section{Journals}

Édition électronique

URL : http://journals.openedition.org/activites/2705

DOI : 10.4000/activites.2705

ISSN : $1765-2723$

\section{Éditeur}

ARPACT - Association Recherches et Pratiques sur les ACTivités

\section{Référence électronique}

Esther González-Martínez et Cécile Petitjean, « Le rire cordial dans les demandes téléphoniques par de jeunes infirmières à l'hôpital », Activités [En ligne], 13-1 | 2016, mis en ligne le 15 avril 2016, consulté le 19 avril 2019. URL : http://journals.openedition.org/activites/2705 ; DOI : 10.4000/activites.2705

Ce document a été généré automatiquement le 19 avril 2019

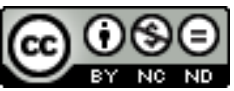

Activités est mis à disposition selon les termes de la licence Creative Commons Attribution - Pas d'Utilisation Commerciale - Pas de Modification 4.0 International. 


\title{
Le rire cordial dans les demandes téléphoniques par de jeunes infirmières à l'hôpital
}

Cordial laughter in young nurses' telephone requests at the hospital

\author{
Esther González-Martínez et Cécile Petitjean
}

\section{NOTE DE L'ÉDITEUR}

Article soumis le 4 février 2015, accepté le 8 décembre 2015.

\section{Introduction}

1 Strauss, Fagerhaugh, Suczek et Wiener (1985) ont examiné le travail à l'hôpital en mettant en évidence des dimensions auparavant peu reconnues, dont le «travail d'articulation » qui consiste à mettre en cohérence des faisceaux de tâches très divers participant à la prise en charge du patient. Les infirmières contribuent de manière centrale à cette articulation de par leur position d'intermédiaires entre les patients et l'institution médicale (Acker, 2005; Grosjean, \& Lacoste, 1999). L'académisation et la professionnalisation de l'activité infirmière, l'ajustement de celle-ci à des logiques gestionnaires, ainsi que la diversification et l'augmentation du nombre d'intervenants auprès des patients, ont accentué l'importance de la coordination infirmière des soins (Allen, 2001 ; Chaska, 2001). Dans un environnement caractérisé par la mobilité et la distribution spatiale des intervenants (Bardram, \& Bossen, 2005), ainsi que par la pression relative au respect du timing (Pouchelle, 1998 ; Raveyre, \& Ughetto, 2006), les infirmières utilisent souvent des moyens de communication à distance, principalement le téléphone, pour contacter d'autres membres du personnel hospitalier (Vaucher, \& GonzálezMartínez, 2015 ; Woloshynowych, Davis, Brown, \& Vincent, 2007). 
2 Cet article porte sur le rôle des rires brefs et ténus produits par de jeunes infirmières lors de leurs demandes téléphoniques à d'autres collaborateurs de l'hôpital. Il est issu d'une recherche sur la coordination téléphonique des soins par des infirmières se trouvant dans leur première année d'engagement à l'hôpital après l'obtention de leur diplôme $\mathrm{e}^{1}$. Lors de cette période cruciale pour leur intégration professionnelle (Wangensteen, Johansson, \& Nordström, 2008), ces jeunes infirmières apprennent à réaliser une activité téléphonique à laquelle il est difficile de les préparer sur les lieux de formation, ne serait-ce qu'en raison de la diversité des interlocuteurs et des situations rencontrées. Elles sont invitées à communiquer de manière rapide, synthétique, claire et fiable (Barenfanger, Sautter, Lang, Collins, Hacek, \& Peterson, 2004) sur des objets et avec des collègues qu'elles sont encore en train de découvrir. Notre recherche a eu lieu dans un hôpital de soins aigus en Suisse romande, au sein duquel nous avons mené un travail de terrain et recueilli un corpus d'appels téléphoniques réalisés ou reçus par trois jeunes infirmières du service de Chirurgie en contact avec du personnel de 14 services différents. L'analyse conversationnelle (Sacks, Schegloff, \& Jefferson, 1974) de ces appels nous a permis de constater qu'ils véhiculent régulièrement une activité de demande (Curl, \& Drew, 2008) initiée par l'infirmière. Celle-ci demande à un médecin de passer examiner un patient dans sa chambre, appelle un transporteur pour qu'il amène un patient au bloc opératoire ou sollicite d'une laborantine la réalisation d'une analyse en urgence ${ }^{2}$. Ces demandes sont adressées à des interlocuteurs sollicités de manière répétée ; par ailleurs, ces demandes sont fréquemment émaillées de difficultés, en partie inhérentes à l'activité téléphonique en tant que telle, en cela qu'elles font irruption dans le travail de la personne appelée par l'infirmière, risquant par là même de la déranger. Malgré ces difficultés, ces demandes ne sont jamais rejetées - elles sont tout au plus réorientées vers d'autres personnes ou objets - ; leur enjeu premier n'est donc pas leur satisfaction mais le fait même de parvenir à les produire de manière fluide, rapide et sans accrocs, tant au niveau pratique que relationnel.

3 Pour produire leurs demandes téléphoniques, les jeunes infirmières se basent sur une multitude de ressources qui sont mobilisées avant même le début de l'appel, telles que des connaissances sur les personnes à appeler en fonction de la question à traiter, la manière de les contacter ou encore les moments auxquels elles sont disponibles. Ces ressources ne trouvent pas leur origine dans une formation spécifique à l'activité téléphonique (inexistante à l'hôpital) mais sont le fruit de l'observation des collègues, de discussions avec ces derniers ainsi que de la propre expérience de la jeune infirmière avec l'utilisation du téléphone. Au cours de l'appel, l'infirmière mobilise encore d'autres ressources qui agissent, par exemple, sur le choix du format de la demande le plus approprié à son objet, ou des éléments à mettre en avant pour expliciter celui-ci (González-Martínez, Markaki, \& Bovey, 2016 ; Petitjean, Sterie, Vaucher, \& GonzálezMartínez, 2015 ; Sterie, 2015). Dans cet article, nous concentrerons notre attention sur une autre de ces ressources: des rires brefs et ténus produits unilatéralement par l'infirmière.

L'importance de l'humour lors des échanges de travail à l'hôpital est un phénomène bien documenté, en lien avec la gestion de situations éprouvantes, le maintien de l'attention sur un objet traité en commun, la redéfinition d'asymétries relationnelles et la cohésion des équipes (Goffman, 1961; Loriol, 2013; Zolesio, 2013). Lors de nos observations à l'hôpital, nous avons constaté que les infirmières expérimentées mobilisaient fréquemment l'humour lors de leurs conversations téléphoniques avec d'autres membres 
du personnel. En examinant notre corpus d'enregistrements, nous avons par contre noté que, si les jeunes infirmières rient très fréquemment lors des appels téléphoniques de demande, seule une minorité de ces rires est associée à des activités humoristiques telles qu'une blague ou une moquerie. La plupart des rires interviennent alors que des contingences rendent l'activité de demande problématique pour une raison ou pour une autre : l'infirmière dérange un supérieur, ignore si sa demande est vraiment nécessaire ; l'appelé n'est pas responsable du traitement de celle-ci ou accepte de la satisfaire tout en étant surchargé. À ce moment, l'infirmière produit des particules de rire (Glenn, 2003), brèves et ténues, qui ne sont ni précédées ni suivies de rire par l'interlocuteur. Ces rires sont parfois associés à la production de tours de parole produits par l'infirmière avec une voix souriante (Haakana, 2010) ainsi qu'à des ressources langagières telles que des formules de politesse. Nous montrerons que par ces rires l'infirmière minimise - et donc reconnait - des aspects problématiques de la demande ou de son traitement, en introduisant une touche de cordialité dans ses interventions, la cordialité se définissant comme une dimension caractéristique de la sociabilité, laissant transparaître les individualités sans les approfondir, mettant en avant la jovialité et aplanissant ce qui pourrait l'entacher (Simmel, 1981[1917]). Ces dimensions pratiques et relationnelles sont fortement inter reliées, en cela qu'elles concourent conjointement à l'avancement de l'activité, ce vers quoi s'oriente précisément l'interlocuteur de l'infirmière dans sa réponse.

5 La construction de l'identité professionnelle et d'une valeur positive de soi (face) est certainement un enjeu important pour la jeune infirmière diplômée (o'Shea, \& Kelly, 2007). Si les actions accomplies par les interlocuteurs de nos appels téléphoniques ne sont pas sans lien avec la construction de l'identité et la gestion des faces, ces derniers se montrent principalement concernés par la réalisation de la demande. Il s'agit donc de suivre les orientations manifestées par les interlocuteurs (Schegloff, 1992), donc leur centration sur l'activité de demande, ce qui délimite de manière stricte la portée de notre entreprise. Cet article contribue ainsi à l'analyse conversationnelle du rire (Glenn, 2003 ; Glenn, \& Holt, 2013) associé à des activités de demande en contexte professionnel. Il se veut par ailleurs utile à la réflexion sur les compétences interactionnelles (Hall, \& Pekarek Doehler, 2011) mobilisées par la jeune infirmière dans son nouvel environnement de travail. Il met aussi en avant des phénomènes qui pourraient in fine être en rapport avec la construction interactionnelle d'identités professionnelles (Greco, Mondada, \& Renaud, 2014) sans pour autant s'attaquer frontalement à cette question.

\section{Appels téléphoniques à l'hôpital, demandes et rire}

Dès ses origines, l'analyse conversationnelle est étroitement liée à l'étude d'appels téléphoniques en milieu clinique (Sacks, 1966). La recherche s'est toutefois concentrée sur des appels entre les professionnels et leurs patients (cf. Pilnick, Hindmarsh, \& Gill, 2009). Il en va de même de la littérature médicale, qui porte principalement sur des interventions téléphoniques à destination des patients (Greenberg, 2009; Wahlberg, 2004). Lorsque cette littérature s'intéresse à des appels entre des professionnels, elle poursuit le développement de nouveaux protocoles de transmission d'informations (Barenfanger et al., 2004 ; Wakefield, Wakefield, Despins, Brandt, Davis, et al., 2012) ou l'évaluation de nouveaux dispositifs technologiques (Hansen, \& Bardram, 2007). Elle cherche à quantifier l'utilisation du téléphone et ses caractéristiques - en termes 
d'interlocuteurs, moment et durée des appels, leurs raisons et leurs issues (Bernstam, Pancheri, Johnson, Johnson, Thomas, \& Turley, 2007) - mais ne propose pas d'analyse fine de l'organisation des conversations.

7 L'analyse conversationnelle traite les demandes comme des activités réalisées au travers d'une paire adjacente, deux tours de parole contigus et inter reliés, formant une séquence de type demande+acceptation/rejet (Sacks et al., 1974) ${ }^{3}$. Le positionnement séquentiel et le formatage des tours en première et en deuxième positions répondent à des préférences conversationnelles qui favorisent la contiguïté et l'accord (Sacks, 1987) et contribuent à la progressivité de l'activité (Schegloff, 2007). Schegloff (2007) défend le statut non préférentiel des demandes, en tant que premiers éléments de paires adjacentes, lors des conversations ordinaires: des séquences préliminaires (pré préséquences et préséquences) permettraient de devancer les demandes et de minimiser les risques de rejet; les demandes n'apparaîtraient qu'une fois la conversation bien engagée et seraient accompagnées d'explications et de justifications. Lee (2009) et Lindström (2005) nous rendent toutefois attentifs au fait que les demandes constituent la raison d'être des rapports de service au cours desquels elles ne manifestent pas les traits caractéristiques des actions non préférentielles (c.-à-d. réponses retardées, mitigées et/ou suivies de justifications). Les travaux en analyse conversationnelle ont également mis en évidence le rapport entre le format du tour produisant la demande et l'orientation du locuteur envers, d'une part, sa légitimité à réaliser la demande et, d'autre part, la faculté de son interlocuteur à y répondre (Curl, \& Drew, 2008 ; Lindström, 2005). Les locuteurs se servent de séquences insérées entre la demande et son acceptation/refus pour demander/donner des précisions par rapport à la demande, évaluer sa légitimité et la façonner en fonction des possibilités du destinataire à la satisfaire (Lee, 2009 ; Whalen, Zimmerman, \& Whalen, 1988). Enfin, s'appuyant sur Sacks (1992) qui montre que l'action de demander peut être implémentée au travers de formats et de séquences propres à d'autres types d'action, Gill (2005) signale qu'une demande peut être réalisée et satisfaite lors d'une interaction médecin-patient sans qu'il soit possible d'identifier un tour de parole en particulier grâce auquel elle aurait été formulée.

8 Parallèlement à la mise à jour de la complexité de l'activité de demande, la recherche en analyse conversationnelle révèle par ailleurs que le rire, loin de son image de réaction physiologique automatique, fait l'objet d'une modulation interactionnelle extrêmement subtile et constitue une ressource précieuse pour réaliser de très nombreuses actions du fait de sa plasticité, de son caractère non propositionnel et de ses grandes possibilités d'insertion dans l'échange (Glenn, \& Holt, 2013). Explorant le rire produit en dehors de situations ouvertement humoristiques, des travaux montrent qu'il participe régulièrement à la production et à la gestion d'activités délicates ou problématiques (Glenn, 2013 ; Osvaldsson, 2004 ; Petitjean, \& González-Martínez, 2015). Concernant les interactions médicales, Haakana (2010) montre que, lorsque le patient rit en présentant la raison de la visite, il indique que celle-ci est atypique ou surprenante et montre qu'il en est conscient. Ticca (2013) montre quant à elle que la mère, en se limitant à rire, refuse de répondre à une question du médecin qui véhicule une critique sur les soins apportés à son bébé.

9 Toujours en analyse conversationnelle, les auteurs insistent sur le fait que le rire est également une ressource relationnelle fortement reliée aux buts pratiques de l'échange (Glenn, \& Holt, 2013)4. Il permet par exemple de moduler dans quelle mesure l'interaction s'oriente vers l'établissement d'un rapport proche avec l'interlocuteur (person oriented) ou 
vers l'accomplissement d'une tâche (task oriented) (Houtkoop-Steenstra, 1997). Ainsi, Lavin et Maynard (2001) considèrent que le rire, les quasi-rires (quasi-laughter) et la voix souriante sont des ressources mobilisées lors d'entretiens téléphoniques de sondage pour gérer la tension entre la standardisation de l'échange et sa personnalisation dans le but de faire avancer l'activité. Nguyen (2007) considère que le rire et le sourire lors d'interactions en classe servent à concilier les exigences de l'enseignement - dont la production d'instructions et de corrections - avec le maintien d'une relation proche et cordiale entre l'enseignant et ses élèves. Jefferson, Sacks et Schegloff (1987) montrent que le rire constitue un signe d'appréciation d'un récit, qui a des implications autant dans l'avancement du récit que dans l'établissement d'une relation d'intimité. L'analyse conversationnelle a exploré aussi bien des échanges purement fonctionnels que la considération distante des échanges de politesse, tout comme des interactions caractérisées par la compréhension empathique ou bien le partage émotionnel propre à l'expression de sympathie (Hewitt, McCloughan, \& McKinstry, 2009; Kasper, 2009; Pudlinsky, 2005; Ruusuvuori, 2007) ${ }^{5}$. Au fil de ces travaux, la cordialité (Simmel, 1981 [1917]) apparaît comme une modalité relationnelle intermédiaire - exigeant plus de proximité interpersonnelle que le rapport de politesse mais moins que n'en requiert l'échange empathique - que le rire peut contribuer à mettre en place lorsqu'il est mobilisé de manière spécifique, comme nous le verrons dans la suite de cet article.

\section{Données et approche analytique}

Cet article repose sur l'analyse d'un corpus de 374 appels téléphoniques, enregistrés pendant une durée de six mois, entre trois jeunes infirmières - deux femmes et un homme - et d'autres membres du personnel d'un hôpital en Suisse romande. Au moment de l'enregistrement des appels, les infirmières se trouvent dans leur première année d'engagement à l'hôpital après l'obtention de leurs diplômes et travaillent dans deux unités différentes d'un même service de Chirurgie. Les appels enregistrés ont été réalisés ou reçus par ces trois infirmières en utilisant le téléphone fixe du bureau des infirmières de leur unité. Leurs interlocuteurs travaillent dans 14 services différents (Chirurgie, Salle de réveil, Transport patients, Laboratoire, Pharmacie, etc.) et utilisent différents types d'appareils (portables et fixes, personnels et d'équipe). Le recueil des enregistrements a été accompagné d'un travail ethnographique à l'hôpital, dans les unités des trois infirmières, ainsi que dans les services où travaillent leurs principaux interlocuteurs. Les appels durent en moyenne 56 secondes y inclues les sonneries téléphoniques.

11 Nous avons transcrit les 374 appels de notre corpus selon les conventions développées par Jefferson (2004). Nous avons ensuite réuni dans une collection tous les extraits incluant des phénomènes de rire et/ou de voix souriante $(n=251)$. Dans cette collection, nous avons identifié 30 extraits dans lesquels est mobilisé le rire infirmier cordial, unilatéral et minimal, qui constitue l'objet de cet article. L'analyse conversationnelle (Sacks et al., 1974) de ces extraits permet d'appréhender les liens entre l'organisation séquentielle de la conversation, le rire et la production de l'activité de demande. Il s'agit d'une entreprise descriptive qui rend compte de manière séquentielle et détaillée de ce que les interlocuteurs font en se parlant (leurs actions) et de la manière de l'accomplir (leurs pratiques) (Clayman, \& Gill, 2004). 


\section{Organisation des appels de demande, contingences problématiques et rire}

12 Les appels de notre corpus présentent généralement la structure standard en trois phases: ouverture, partie centrale et clôture (Schegloff, 1986). À chaque phase, les interlocuteurs réalisent des actions qui contribuent à l'activité de demande. Ainsi, l'ouverture permet de vérifier, ou non, la disponibilité de l'appelé et l'adéquation entre les interlocuteurs - par exemple, la légitimité d'une infirmière $\mathrm{X}$ à contacter un médecin $\mathrm{Y}$ - indispensables à la satisfaction de la demande à venir. Dans la phase centrale, l'infirmière introduit sa demande et reçoit la réponse de l'appelé; tous les deux doivent souvent produire des actions complémentaires nécessaires au traitement de la demande et se mettre d'accord sur la manière de la satisfaire. La clôture de l'appel est une phase également orientée vers la demande. Par exemple, les interlocuteurs y produisent des remerciements qui rendent la demande reconnaissable comme telle et manifestent le fait qu'elle a été traitée de manière positive.

13 Nous avons identifié des pratiques de rire et/ou de voix souriante dans les trois phases de ces appels, en lien avec des contingences de l'activité de demande rendant celle-ci problématique. Pour cet article, nous nous sommes concentrées sur des moments où l'infirmière produit des rires brefs et ténus qui ne sont ni précédés ni suivis de rire par l'interlocuteur. Ces rires sont parfois associés à la production par l'infirmière de tours de parole caractérisés par une voix souriante et à l'utilisation de ressources langagières telles que des formules de politesse. Ces rires interviennent dans différentes phases des appels, dans des environnements actionnels différents mais aussi à différents moments dans la production des tours de parole de l'infirmière, et même parfois en absence de toute verbalisation de sa part. Le nombre de particules de rire est variable, tout comme la qualité de la voyelle de support, qui est parfois absente. Ces rires ont toutefois en commun de minimiser - et donc de reconnaitre - ce qu'il pourrait y avoir de problématique dans la demande ou dans son traitement, et ce en introduisant une touche de cordialité dans les interventions de l'infirmière. Les fonctions pratique et relationnelle du rire se combinent pour faire progresser l'activité, ce vers quoi l'interlocuteur s'oriente dans sa réponse. En effet, l'interlocuteur ne produit ni de rires ni de signes d'une cordialité particulière, mais outrepasse ce qui pourrait faire obstacle à l'avancement de l'activité. Nous nous concentrons donc sur des cas où le rire est minimal et unilatéral. Loin de contredire la fonction que nous attribuons aux rires, cette observation vient la confirmer. L'échange reste focalisé sur l'activité pratique que les deux participants doivent conjointement mener à terme, l'infirmière apportant le supplément de cordialité participant au travail de minimisation des problèmes, incarnant en cela sa position de partie demandeuse et de jeune collègue en cours d'intégration dans son nouvel environnement professionnel.

14 Ceci posé, les demandes téléphoniques exhibent des configurations très différentes en ce qui concerne le rire, qui est parfois tout simplement absent alors que l'activité témoigne de contingences problématiques. Dans ces cas, les interlocuteurs ne font pas d'efforts particuliers pour minimiser les dimensions problématiques de l'activité (sans que cela ne conduise pour autant au rejet de la demande) ou mobilisent à cette fin d'autres ressources que le rire. Les jeunes infirmières peuvent par exemple formuler leurs demandes de manière particulièrement ouverte, réduisant leur force d'imposition, ou bien solliciter la 
compréhension de l'interlocuteur en se présentant comme des apprenantes. Dans d'autres appels, le rire vient minimiser des aspects problématiques des demandes mais les deux interlocuteurs produisent des rires partagés et prolongés. Le lecteur comprendra l'impossibilité de traiter ici de tous ces cas de figure, tout comme de faire des comparaisons avec les appels d'infirmières expérimentées pour lesquels des enregistrements ne sont pas disponibles. Dans cet article, nous nous concentrerons donc sur le rire cordial, unilatéral et minimal, de la jeune infirmière. Cette pratique sera décrite au travers de l'analyse de cinq extraits correspondant aux trois phases ouverture, partie centrale et clôture - d'appels de demande réalisés par Tim, le jeune infirmier ayant participé à notre étude ${ }^{6}$.

\subsection{Rire lors de l'ouverture de l'appel}

15 La phase d'ouverture de l'appel est soumise à de multiples contingences problématiques qui peuvent potentiellement avoir de l'importance pour la demande à venir. Par exemple, l'appel est adressé à un interlocuteur inhabituel, à une heure particulièrement tardive et/ ou à un appelé qui manifeste immédiatement son manque de disponibilité. Nous avons observé que des rires brefs et ténus sont régulièrement associés à ce type de contingences propres à une phase d'ouverture clé dans le lancement de l'activité. Nous analyserons ici un extrait où l'infirmier rit, en demandant qu'on l'excuse, lors de l'ouverture d'un appel potentiellement problématique.

Dans l'extrait 1 ci-dessous, Tim appelle une médecin assistante de son service, Ida. L'appel a lieu à 6 heures du matin : Tim assure donc le service de nuit et Ida est de garde. Lors de l'ouverture de l'appel (1-8), Tim introduit une particule de rire dans l'expression « excuse-moi » à la ligne $7^{7}$.

Extrait $1:$ Rire lors de l'ouverture - appel $\mathrm{C} 176$.

Excerpt 1: Laughter in the opening - call C176

\section{Ext rai t 1: C176}

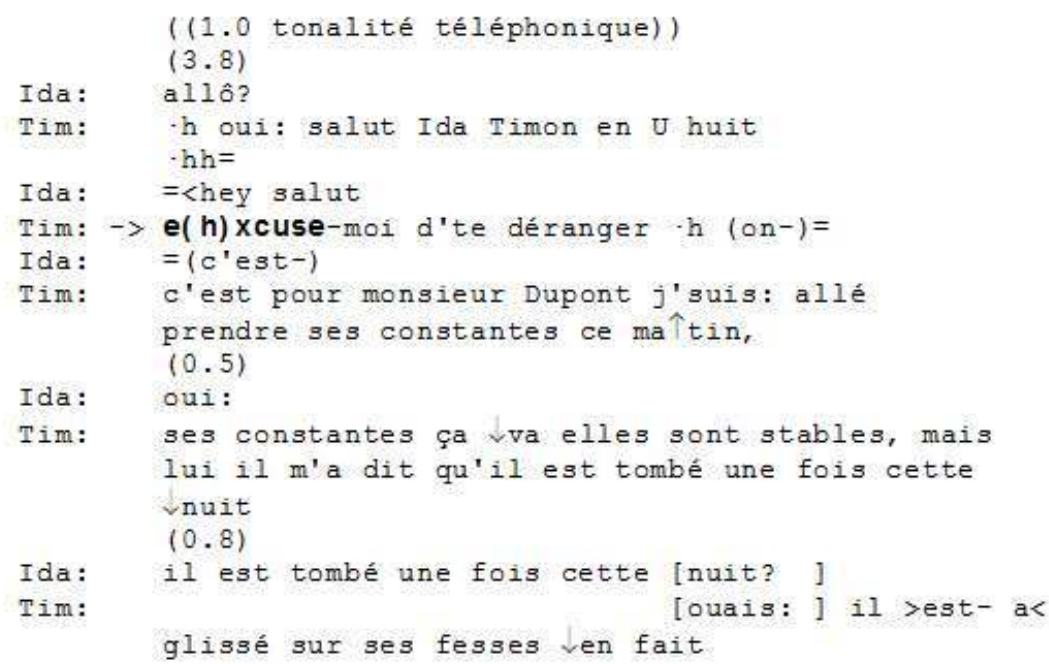

17 L'extrait 1 ci-dessus comprend tout d'abord une séquence d'interpellation-réponse : le téléphone sonne et Ida y répond avec l'expression générique «allô?» (3). Tim réagit à cette réponse par un « oui » élongé en début de tour suivi d'une salutation familière et du prénom de la médecin (4); le tout vaut reconnaissance de la médecin. Ensuite, Tim s'auto- 
identifie par son prénom suivi du nom abrégé de son unité hospitalière d'appartenance. Alors que Tim s'apprête à poursuivre son tour, Ida produit des expressions familières marquant l'identification de son interlocuteur et le saluant (6), le « hey » initial indiquant un accueil quelque peu surpris de l'appel de Tim. Cette séquence, composée de salutations familières, d'identifications et de reconnaissances par le prénom, met en place un échange entre collègues proches. Tim poursuit en s'excusant de déranger Ida (7), s'orientant probablement vers le caractère matinal de l'appel, le caractère très prenant de l'activité de garde et la surprise manifestée par Ida ${ }^{8}$. Tim insère une particule de rire au début du mot «excuse» qui introduit de la légèreté dans la situation, invitant à minimiser l'importance de l'irruption téléphonique. Ce rire ajoute aux excuses une touche de cordialité, dépassant en cela la simple formule de politesse ${ }^{9}$. Ida répond en soutenant l'avancement de l'activité avec ce qui pourrait être le début d'une invitation afin que Tim présente la raison de l'appel («c'est», 8), abandonnée dès que Tim s'y engage lui-même («c'est», 9). Celui-ci expose alors le cas d'un patient qui requiert l'intervention de la médecin.

Dans cet extrait, une particule de rire vient ponctuer le mot «excuse » produit par l'infirmier dans l'ouverture de l'appel. La formule de politesse montre que l'infirmier reconnaît que l'appel pourrait potentiellement déranger la médecin et exprime de la considération à son égard. Le rire ajoute une touche de cordialité à l'intervention de l'infirmier qui invite à minimiser subtilement ce que celle-ci pourrait avoir de problématique. Ce rire n'est pas formaté de manière à inviter l'interlocutrice à rire à son tour ou à se montrer particulièrement affable. La réponse de la médecin consiste d'ailleurs à soutenir l'avancement de l'activité, tandis que Tim fait suivre rapidement ses excuses par la présentation d'une situation médicale qui véhicule une demande d'intervention de l'interlocuteur. Il est à remarquer que Tim présente toutefois cette situation comme n'étant pas particulièrement inquiétante : priorité donnée à la référence aux constantes normales du patient (9-13), référence à la chute du patient par le biais d'un discours rapporté («lui il m'a dit qu'il est tombé », 14), puis description plutôt « cocasse » de l'incident («il a glissé sur ses fesses », 18-19). L'excuse riante de Tim à la ligne 7 pourrait d'ailleurs être en rapport avec la construction de cet incident présenté comme ne justifiant pas d'alarme ; une raison supplémentaire, en sus de l'heure matinale, pour laquelle l'appel aurait un caractère quelque peu problématique, à minimiser.

\subsection{Rire lors de la production et le traitement de la demande}

19 L'appel entre dans sa phase centrale lorsque l'infirmière commence à formuler sa demande. La production de cette action peut alors être rendue problématique par de nombreuses contingences - par exemple, l'infirmière se trompe quant au nom du patient à assister ou du médicament à lui administrer. Leur manifestation s'accompagne de rires brefs et ténus. Il arrive aussi que ces rires interviennent alors que la demande est présentée comme étant en elle-même problématique. Nous analyserons deux extraits en témoignant.

Dans l'extrait 2 ci-dessous, Tim appelle le numéro principal des assistantes hôtelières (des membres du personnel de la cuisine de l'hôpital) et commande un repas pour une patiente. À l'heure où l'appel est réalisé, il est trop tard pour faire la demande via le système informatique (le Logimen) que les infirmières utilisent normalement pour la 
commande des repas. Tim produit une particule de rire articulée au mot « commander » à la ligne 6.

Extrait 2 : Rire lors du tour de demande - appel $\mathrm{C} 137$.

Excerpt 2: Laughter during the request turn - call C137

\section{Ext rai t 2: C137}

1

2

3 Lil:

Tim:

Lil:

Tim: -

Iil:

Tim:

Lil:

Tim:

Lil:
((1.0 tonalité téléphonique))

$(0.7)$

'h oui Liliana?

$<\cdot h$ oui: salut Timon en $\mathrm{U}$ huit $\cdot \mathrm{h}=$

$\Rightarrow \uparrow$ salut $<$

$>j$ 'avais besoin de commande( $h) r$ un re $\downarrow$ pas

en fait en plus

$(0.3)$

h ou[i: quoi? ]

[j'ai un pro]blème avec logimen j'arri-

>on arrive< pas à rentrer la dame hh

$(0.5)$

d'a $[\mathrm{cco}: \mathrm{rd}$

]

[>ça $s$ 'rait< un] $r$ 'pas nor $\downarrow_{\text {mal }}$ en $\downarrow_{\text {fait }} h$ h

$>\downarrow$ ouais< <tu m'envoies juste un bon cui $\uparrow$ sine et

ça sera [dans l'cha]rriot=

Dans cet extrait, Tim entre dans la phase centrale de l'appel en exprimant un besoin qui véhicule une demande (6). Le formatage du tour s'oriente vers le caractère délicat de cette action: Tim exprime un besoin personnel et indique qu'il s'agit d'une commande supplémentaire, qui s'ajoute à d'autres, précédemment réalisées, et qui aurait donc été négligée (utilisation de l'imparfait "avais », 6 et de l'expression « en fait en plus », 7). Lors des passages que les assistantes hôtelières font dans les unités, Tim a probablement manqué de transmettre cette demande à l'assistante hôtelière, Lil. Tim insère une particule de rire dans le mot désignant l'élément focal de la demande, «commander », et indique ce faisant le caractère singulier de celle-ci. Le rire ajoute une touche de cordialité et invite à minimiser l'aspect problématique de la demande pour la traiter de manière favorable. La réponse n'est pas immédiate et Tim initie une explication de la raison pour laquelle la demande n'a pas été faite en premier lieu à l'aide du Logimen (10-11), ce qui montre son orientation vers sa dimension problématique. Lil est toutefois déjà en train de lui demander de quel type de repas il s'agit, contribuant ainsi à l'avancement de l'activité.

Dans l'extrait 3 ci-dessous, le rire intervient non pas lors de la production du tour de demande, comme dans l'extrait 2, mais à la fin de celui-ci. Lors d'un appel au numéro principal du laboratoire de l'hôpital, Tim produit un récit des opérations en rapport avec une analyse sanguine, qu'il conclut par un rire (« huhh »,11). 
Extrait 3 : Rire à la fin du tour de demande - appel $\mathrm{C} 173$.

Excerpt 3: Laughter at the end of the request turn - call C173

\section{Extrai t 3: $\mathrm{C173}$}

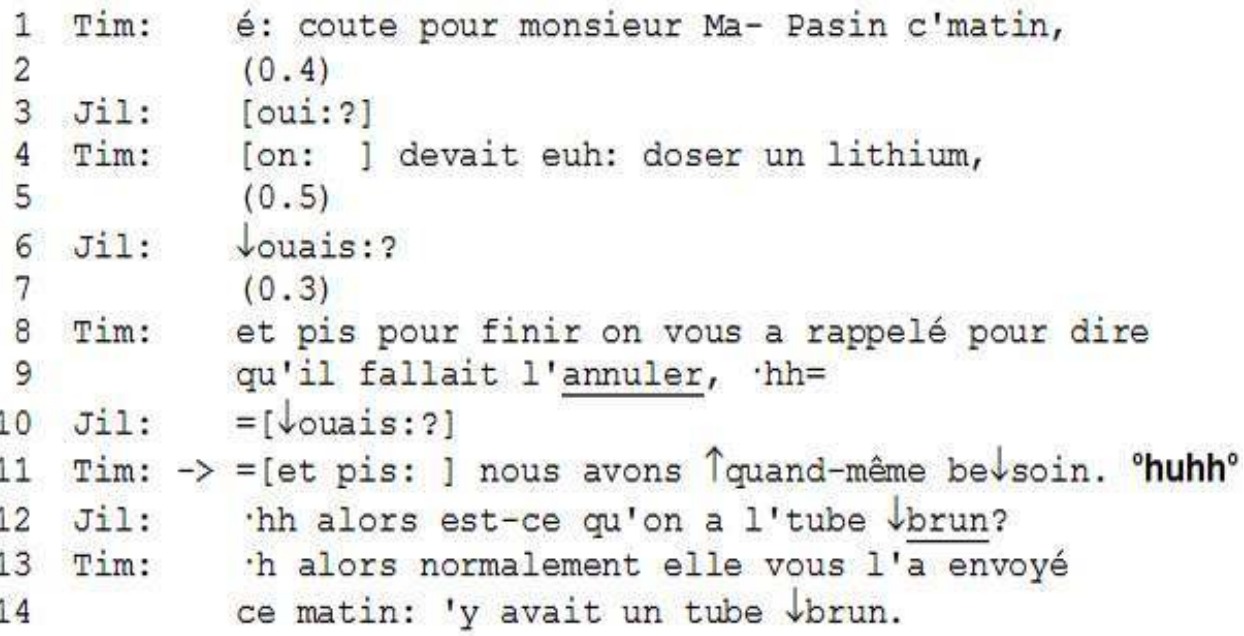

Dans cet extrait, Tim s'engage dans la phase centrale de l'appel en laissant entendre avec un «écoute» hésitant, produit en deux temps - une situation quelque peu compliquée requérant l'attention de la laborantine, Jill. Il indique de quel patient il s'agit puis expose pas à pas les opérations effectuées en rapport avec une analyse sanguine. Le tour de parole de Tim est formé d'une succession d'unités à intonation progressive $(1,4$, 8-9) laissant entendre un récit en cours de production. Après la première unité, Jil produit un «oui » continuateur (3). Après la deuxième et la troisième unité, ses microinterventions prennent la forme de « ouais » $(6,10)$ sollicitant une suite qu'ils anticipent comme étant problématique (ton de voix plus grave, allongement vocalique, intonation interrogative). La dernière unité du récit de Tim (11) introduit une demande d'analyse sanguine qui avait été précédemment annulée. Dans un contexte professionnel où le temps est une ressource précieuse, faire une demande une première fois, l'annuler pour ensuite la réintroduire est clairement problématique. D'ailleurs, nous notons qu'en utilisant les pronoms sujets "on » et «ils», Tim évite de se présenter dans son récit comme agent des actions rapportées alors que c'est lui-même qui a appelé précédemment le laboratoire pour annuler l'analyse (cf. extrait 5 ci-dessous). Tim fait suivre la conclusion de son récit d'un rire bref produit doucement («huhh»,11) exprimant une cordialité qui invite à minimiser la dimension problématique de la situation rapportée. La laborantine répond à la demande en posant une question qui initie son traitement (12), œuvrant ainsi à l'avancement de l'activité.

Les extraits ci-dessus nous montrent deux occurrences de rire au moment de la production de la demande, dans deux positions différentes (à l'intérieur et à la fin du tour de l'infirmier). Ils contribuent à adoucir des demandes problématiques qui s'écartent des procédures habituelles. Le rire, bref et ténu, n'invite nullement à une réception humoristique des demandes et décourage également, à l'autre bout du spectre possible des réactions, la production d'une plainte de la part de l'interlocuteur. En riant, l'infirmier reconnaît le caractère singulier de sa demande, invite son interlocuteur à en minimiser l'importance et à s'orienter à son tour vers la cordialité que le rire introduit dans son intervention. Dans les deux cas, la réponse de l'interlocuteur n'est ni de rire, ni 
de se plaindre, ni de signaler d'une façon ou d'une autre qu'il y a eu un problème, mais de faire le nécessaire pour traiter la demande.

Le traitement des demandes est également soumis à de multiples contingences qui font obstacle à l'avancement de l'activité, face auxquelles les infirmières réagissent souvent en produisant des tours de parole avec des particules de rire et/ou, parfois, une voix souriante. Nous analysons ci-après un extrait dans lequel la demande ne peut pas être satisfaite immédiatement et où l'infirmier rassure son destinataire sur le caractère non problématique de cet ajournement.

Dans l'extrait 4 ci-dessous, Tim appelle le numéro central du laboratoire de l'hôpital. Il est minuit et, à cette heure-ci, seules des analyses d'urgence sont réalisées. Suite à la salutation de Ria, la laborantine, Tim présente la raison de son appel en esquissant une demande de résultat d'une analyse sanguine (2-3) qui aurait donc été commandée au préalable. La laborantine, Ria, n'est pas en mesure de donner immédiatement l'information demandée et déclare son intention de se déplacer vers la machine effectuant l'analyse afin de l'obtenir (7). Tim manifeste qu'il apprécie le geste puis rit («c'est gentil m(h) hum », 9).

Extrait $4:$ Rire lors de la gestion de la demande - appel $C 133$.

Excerpt 4: Laughter while dealing with the request - call C133

\section{Ext rait 4: $\mathrm{C133}$}

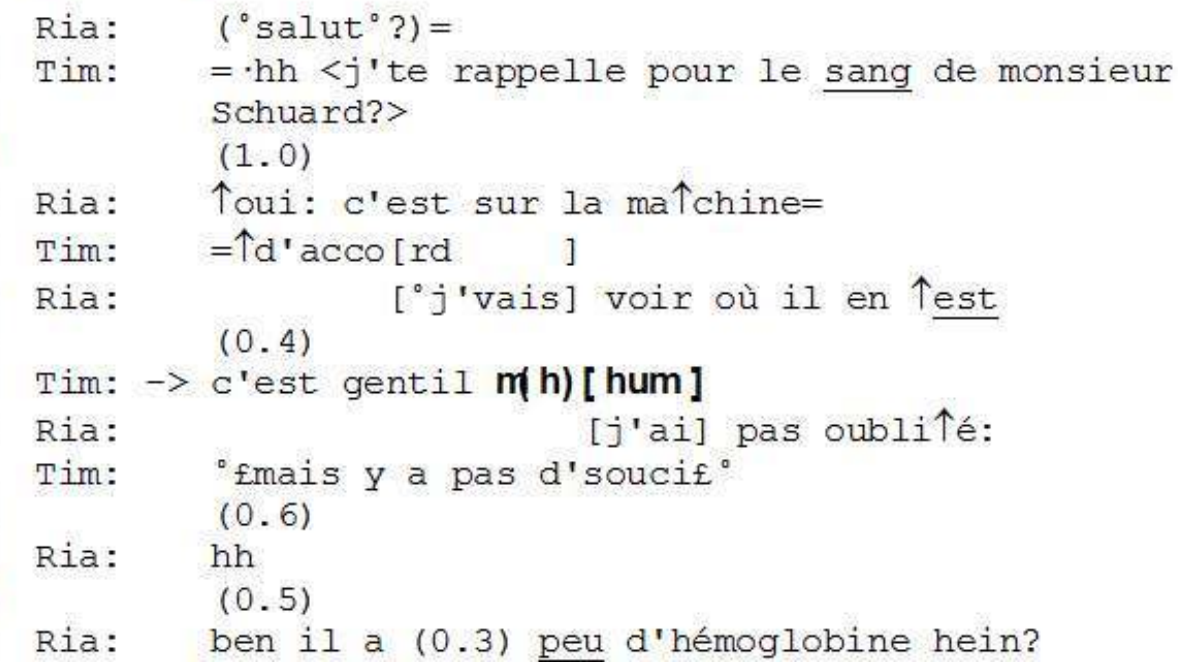

Aux lignes 2-3, Tim indique qu'il est en train de rappeler le laboratoire et donne la raison de son appel - «le sang » d'un patient - de manière elliptique. Ce tour de parole évoque un échange précédant au sujet de la même analyse: Tim a sûrement demandé l'analyse par téléphone (comme il est habituel de le faire pour les demandes d'urgence pendant la nuit) et il se peut qu'il ait déjà appelé une première fois pour obtenir le résultat. Par ailleurs, ce tour de parole, produit avec une intonation interrogative, véhicule une demande d'information et projette une réponse. Toutefois, la laborantine reste silencieuse, signalant en cela que l'intervention de Tim est insuffisante ; celui-ci s'abstient pourtant d'ajouter quoi que ce soit (4). Ria finit par produire une information factuelle sur l'avancement de l'analyse ("c'est sur la machine », 5). Son tour explicite l'objet de la demande (le résultat de l'analyse), la problématise (la demande est hâtive par rapport au temps nécessaire pour effectuer l'analyse) et indique la raison pour laquelle cette demande est difficile à satisfaire (l'analyse serait toujours en cours). Sans se 
décontenancer, Tim se limite à recevoir l'information («d'accord», 6) et Ria se déclare sur le point de se déplacer vers la machine d'analyse pour apporter une réponse à la demande (7). Tim apprécie le geste (« c'est gentil ») et produit ensuite un rire bref («m(h) hum », 9). Ce rire introduit de la cordialité dans la formule de politesse ainsi qu'une certaine légèreté dans une situation où couveraient autrement des reproches pour un appel injustifié et l'absence du résultat. Ria s'oriente vers ces éventuels reproches lorsqu'elle ajoute qu'elle n'a pas oublié l'analyse (10). Ici encore, Tim opte pour une réponse qui est doublement habile en cela qu'elle lui permet de minimiser l'importance d'un éventuel problème, que celui-ci soit causé par Ria ou par lui-même. Il rassure la laborantine avec une formule de politesse qui débute avec une particule oppositive ( «mais y a pas d'souci »,11). D'une part, la réponse de Tim évite de reconnaittre qu'il pourrait y avoir quoi que ce soit de problématique dans le fait de rappeler la laborantine. D'autre part, elle témoigne du traitement de la déclaration de la laborantine à la ligne 10 ("j'ai pas oublié ») non pas comme un reproche adressé à Tim mais comme une justification de Ria pour son retard dans l'exécution des analyses. Le tour est produit avec une voix souriante, soulignant ainsi le fait que Tim traite la situation avec cordialité sans lui accorder autrement de l'importance. Dans l'enregistrement, on entend Ria se déplacer et expirer bruyamment (13) puis donner ses impressions sur le résultat de l'analyse en question, ce qui assure l'avancement de l'activité de demande.

Dans les deux premiers extraits de cette partie, les rires analysés étaient en rapport avec le fait de produire une demande problématique. En riant, l'infirmier auteur de la demande invite son destinataire à dépasser le caractère problématique de celle-ci au lieu de formuler, par exemple, un reproche. Dans le troisième extrait, la situation est différente et plus complexe. L'infirmier apparaît comme l'auteur d'une action problématique - faire preuve d'impatience - tout comme son interlocutrice qui n'est pas en mesure de satisfaire immédiatement une demande alors que le contexte de travail exige de la célérité. Il nous paraît intéressant de remarquer que ce n'est pas la laborantine qui rit, retirant ainsi de l'importance à la situation problématique, mais l'infirmier. Ce fait pourrait indiquer qu'il est attendu un effort supplémentaire de la part de la partie demandeuse, de surcroît lorsqu'il s'agit d'une jeune personne en phase d'intégration professionnelle, pour parer ce qui pourrait faire obstacle à l'avancement de son travail. L'infirmier produit tout d'abord des formules de politesse pour montrer son appréciation quant au fait que la laborantine est disposée à satisfaire sa demande, puis pour la rassurer sur le fait qu'il n'accorde pas d'importance au retard dans la réalisation des analyses. De par ses rires et sa voix souriante, l'infirmier montre qu'il prend la situation avec bonne humeur, dans un esprit de cordialité. En agissant ainsi, il évite de s'engager dans un échange sur les justifications défensives produites par la destinataire de la demande, échange qui risquerait de retarder d'autant plus la satisfaction de celle-ci. Le rire ténu évite également un traitement humoristique de la situation, qui aurait le même effet retardateur et vers lequel l'interlocutrice ne semble nullement s'orienter. Il est possible que la voix souriante qui suit le rire permette de continuer à minimiser l'importance de ce qui pourrait poser problème et à introduire de la cordialité, alors que de nouveaux rires risqueraient d'indiquer qu'il y a "quelque chose de drôle" dans les propos échangés, ce qui n'est pas ici le cas ${ }^{10}$. 


\subsection{Rire lors de la résolution de la demande et la clôture de l'appel} l'annulation d'une commande d'analyse effectuée auparavant ${ }^{11}$. La laborantine, Ros, répond positivement et, immédiatement après, l'infirmier rit puis produit un énoncé appréciatif avec une voix souriante («hhu d'accord c'est gentil merci », 12).

Extrait 5 : Rire en recevant la réponse à la demande - appel $C 172$.

Excerpt 5: Laughter while receipting the response to the request - call C172

\section{Ext $r$ ai $t$ 5: $\quad$ c172}

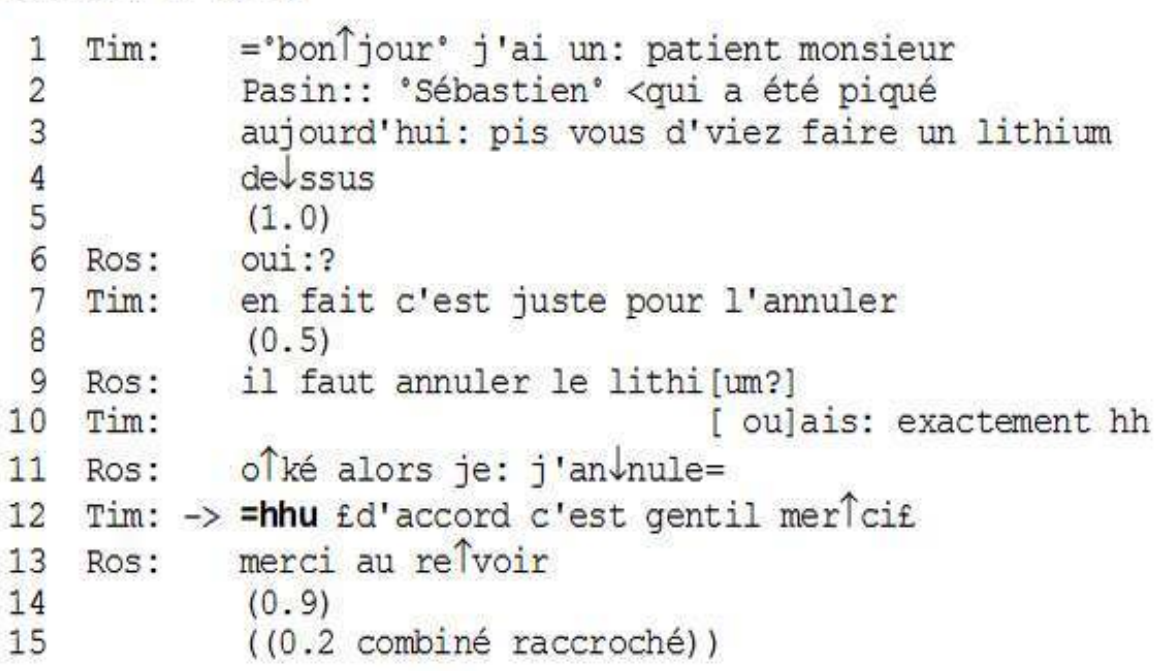

31 À la ligne 1, Tim opère la transition entre l'ouverture de l'appel et sa phase centrale. Il salue la laborantine puis évoque une demande d'analyse, transmise auparavant au laboratoire, pour l'un de ses patients (1-4). Après un silence, Ros l'invite à poursuivre (« oui », 6) et Tim donne la raison de son appel, véhiculant une demande d'annulation de l'analyse précédemment commandée (7). Il s'ensuit une séquence insérée dans laquelle Ros sollicite et obtient une confirmation de la demande (9-10). Ros accepte la confirmation («oké ») et se présente immédiatement disposée à satisfaire la demande («alors je j'annule», 11). Tim enchaîne avec une particule de rire (12) qui accueille favorablement la bonne disposition de Ros. Puis, avec une voix souriante, il exprime son accord avec l'annulation, apprécie la réponse favorable de Ros («c'est gentil») et la remercie (12). Ros remercie Tim à son tour et prend congé de lui (13).

Dans cet extrait, Tim formule une demande qui pourrait être problématique en cela qu'elle consiste à annuler une précédente demande. De ce fait, Tim met un soin particulier à préparer le terrain avant de formuler sa demande : il présente d'abord la 
situation générale puis attend que Ros l'invite à poursuivre. À ce stade, il ne rit pas pour minimiser le caractère problématique de la demande. Il le fait uniquement après que Ros a accepté d'annuler l'analyse, saluant ainsi sa bonne disposition. Le rire précédant les formules de politesse ainsi que la voix souriante avec laquelle celles-ci sont produites ajoutent de la cordialité à l'intervention de Tim. Ils communiquent sa satisfaction quant au traitement de la demande, le faisant apparaitre comme une activité aisée entre collègues de travail, et ce malgré les problèmes rencontrés. Comme dans les autres extraits de cet article, la réponse de l'interlocuteur de l'infirmier consiste à faire progresser l'activité, amenant ici l'appel vers son point final.

\section{Discussion et conclusion}

L'analyse des demandes téléphoniques des jeunes infirmières diplômées est révélatrice de la complexité du travail que celles-ci doivent affronter dès les premiers mois de leur engagement à l'hôpital. L'activité de demande est une composante du "travail d'articulation» (Strauss et al., 1985) des tâches de prise en charge du patient. Elle se retrouve dans de nombreux domaines du travail infirmier, autour d'objets cliniques importants, répondant à des critères exigeants, dont l'intervention de spécialistes en cas de besoin ou la réalisation d'examens et de tests médicaux. Importantes en termes d'enjeux et de places au sein du travail infirmier, les demandes interviennent souvent alors que l'infirmière et son interlocuteur sont déjà engagés dans d'autres activités. Lorsqu'elles sont réalisées rapidement, elles minimisent le risque de dérangement, facilitent l'obtention rapide de ce qui est demandé et préservent un rythme de travail soutenu. Toutefois, alors que l'infirmière se trouve constamment à devoir gérer avec rapidité des demandes très diverses, que ce soit au niveau des moyens de communication employés (les demandes téléphoniques n'en sont qu'une forme) que des interlocuteurs et des objets traités, l'activité de demande est soumise à toutes sortes de contingences problématiques qui retardent son accomplissement : l'interlocuteur est absent ou occupé, n'est plus responsable du type de demande en question ou ne sait pas répondre; l'infirmière manque de précisions pour passer sa commande, laquelle peut parfois se révéler finalement inutile, etc. Tout ceci dans un contexte où les jeunes infirmières sont encore en train de se familiariser avec leurs activités, le fonctionnement de l'hôpital et leurs nouveaux collègues.

Une subtile imbrication d'aspects pratiques et relationnels fait que l'activité professionnelle nécessite de mener à bien un certain nombre de tâches tout en ménageant les rapports avec les collègues (Holmes, \& Stubbe, 2003). Les jeunes infirmières cherchent à établir un équilibre entre ces deux dimensions en mobilisant un ensemble de ressources, parmi lesquelles le choix entre divers formats de demande, plus ou moins directifs, ou une habile sélection des éléments à introduire dans celle-ci (González-Martínez et al., 2016; Petitjean et al., 2015 ; Sterie, 2015). Dans cet article, nous nous sommes concentrées sur une autre de ces ressources, à savoir la production par l'infirmière de rires brefs et ténus à différents moments de l'appel. Ces particules de rire ne sont ni précédées ni suivies de rires par l'interlocuteur de l'infirmière et ne participent pas à des activités humoristiques. Mobilisés de manière particulièrement contrôlée, ces rires, accompagnés parfois de voix souriantes, ajoutent une touche de cordialité à la production et au traitement de la demande, minimisant les difficultés qui peuvent y être associées et œuvrant dans le sens de l'avancement de l'activité. Opérant dans un cluster de 
pratiques, les rires apportent une coloration cordiale à des formules de politesse et à d'autres interventions par lesquelles l'infirmière cherche à mettre de côté ce que la situation pourrait avoir de problématique.

Ces rires brefs et unilatéraux mettent en évidence le travail particulièrement subtil effectué par l'infirmière. Celle-ci introduit une forme de légèreté et de cordialité dans ses interventions, minimisant ainsi d'éventuelles difficultés, ne serait-ce que lorsque l'appel vient déranger l'appelé. Une infirmière qui déploierait tout au long de l'appel une voix souriante, accompagnée éventuellement de petits rires, paraîtrait particulièrement amicale. Des rires plus soutenus pourraient indiquer qu'il y a quelque chose d'humoristique dans la situation rapportée ou dans les propos échangés. Le fait que l'interlocuteur de l'infirmière ne réponde pas aux rires que nous avons analysés par d'autres rires ou d'autres expressions de cordialité souligne sa compréhension de la subtilité du travail effectué par l'infirmière. L'échange reste centré sur l'avancement de l'activité, l'interlocuteur mettant de côté ce qui, d'une manière ou d'une autre, pourrait venir entraver cette finalité. Ceci n'empêche pas le fait que les ressources que nous avons étudiées soient mobilisées dans d'autres situations et d'autres configurations. Par exemple, l'appelé répond au téléphone avec une voix souriante, en montrant qu'il connaît déjà l'identité de l'appelant, et l'infirmière le salue en riant. Ou bien l'infirmière rit en présentant ses difficultés avec un patient et le médecin l'assure de son arrivée immédiate avec une voix souriante. Parfois, l'intégralité de l'appel témoigne d'un ton particulièrement amical que les interlocuteurs mettent en place avec de nombreux rires et des voix souriantes. Dans ces cas, l'infirmière fait plus qu'ajouter une touche de cordialité à son intervention - le cas de figure que nous avons examiné dans cet article mais s'engage dans la co-construction d'un rapport particulier avec son interlocuteur.

Introduire dans la conversation des particules de rire, éventuellement accompagnées de voix souriantes, contribue au travail de «doing being personnal» (Houtkoop-Steenstra, 1997). Avec juste le bon degré d'ouverture, au-delà de la politesse sans aller jusqu'à l'échange amical, les rires brefs et ténus que nous avons examinés laissent transparaître un individu affable derrière le professionnel et créent un environnement propice à la progressivité des activités, sans qu'il ne soit question d'inviter de manière contraignante l'interlocuteur à rire à son tour. Des modes d'échange plutôt autoritaires ou agressifs, ou bien plus désinvoltes et humoristiques, constatés chez les infirmières expérimentées, sont encore hasardeux pour de jeunes infirmières en phase d'intégration. La cordialité sied mieux à la jeune infirmière échangeant avec de nouveaux collègues et correspond à la représentation du caractère moral de l'«ange discipliné » décrit par Strauss (2001 [1966]). La jeune infirmière exerce ici une compétence consistant à "bien prendre » les contingences des demandes téléphoniques, attendant que son interlocuteur fasse de même, sans céder à la plainte ou à la moquerie.

Notre analyse met en évidence la subtilité des pratiques interactionnelles investies dans les activités de la jeune infirmière en phase d'intégration professionnelle. Elle invite à élargir l'étude de l'opérativité du rire en contexte hospitalier, laquelle, actuellement, se concentre essentiellement sur le rire en rapport avec l'humour (Loriol, 2013; Zolesio, 2013). Dans une perspective conversationnelle, il serait nécessaire de passer à l'étude des rires qui nous occupent dans des contextes séquentiels et actionnels bien précis (par exemple le rire dans la production d'excuses ou de remerciements). Il serait également nécessaire d'examiner la manière dont ces actions sont déclinées en fonction des ressources langagières et prosodiques mobilisées, notamment des changements du ton de 
la voix (plus grave ou plus aigu), ainsi que des voix souriantes et/ou des rires plus ou moins forts ou prolongés. Autant d'invitations à poursuivre l'exploration de l'extraordinaire richesse du rire comme ressource interactionnelle lors de la réalisation d'activités professionnelles.

\section{BIBLIOGRAPHIE}

Acker, F. (2005). Les reconfigurations du travail infirmier à l'hôpital. Revue française des affaires sociales, 1(1), 161-181.

Adelswärd, V. (1989). Laughter and dialogue. The social significance of laughter in institutional discourse. Nordic Journal of Linguistics, 12(2), 107-136.

Allen, D. (2001). The Changing Shape of Nursing Practice. The role of Nurses in the Hospital Division of Labour. London: Routledge.

Bardram, J. E., \& Bossen, C. (2005). Mobility work. The spatial dimension of collaboration at a hospital. Computer Supported Cooperative Work, 14(2), 131-160.

Barenfanger, J., Sautter, R. L., Lang, D. L., Collins, S. M., Hacek, D. M., \& Peterson, L. R. (2004). Improving patient safety by repeating (read-back) telephone reports of critical information. American Journal of Clinical Pathology, 121(6), 801-803.

Bernstam, E. V., Pancheri, K. K., Johnson, C. M., Johnson, T. R., Thomas E. J., \& Turley J. P. (2007). Reasons for after-hours calls by hospital floor nurses to on-call physicians. The Joint Commission Journal on Quality and Patient Safety, 33(6), 342-349.

Brown, P., \& Levinson, S. (1987). Politeness. Some Universals in Language Use. Cambridge: Cambridge University Press.

Chaska, N. L. (Ed.) (2001). The Nursing Profession. Tomorrow and Beyond. London: Sage.

Clayman, S. E., \& Gill V. T. (2004). Conversation analysis. In M. Hardy, \& A. Bryman (Eds.). Handbook of Data Analysis (pp. 589-606). London: Sage.

Curl, T. S., \& Drew, P. (2008). Contingency and action. A comparison of two forms of requesting. Research on Language and Social Interaction, 41(2), 129-153.

Gill, V. T. (2005). Patient « demand» for medical interventions. Exerting pressure for an offer in a primary care clinic visit. Research on Language and Social Interaction, 38(4), 451-479.

Glenn, P. (2003). Laughter in Interaction. Cambridge: Cambridge University Press.

Glenn, P. (2013). Interviewees volunteered laughter in employment interviews. A case of « nervous laughter »? In P. Glenn, \& E. Holt (Eds.). Studies of Laughter in Interaction (pp. 255-275). London: Bloomsbury.

Glenn, P., \& Holt, E. (Eds.) (2013). Studies of Laughter in Interaction. London: Bloomsbury.

Goffman, E. (1961). Role distance. In Encounters. Two Studies in the Sociology of Interaction (pp. 83-152). New York: The Bobbs-Merrill Company.

Goffman, E. (1974[1967]). Les rites d'interaction. Paris : Minuit. 
González-Martínez, E., Markaki, V., \& Bovey, F. (2016). Demandes régime dernière minute » lors de conversations téléphoniques infirmière-diététicienne. In K. Stroumza (Ed.). Le langage dans l'analyse des activités professionnelles (pp. 83-108). Genève : Éditions IES.

Greco, L., Mondada L., \& Renaud, P. (Eds.) (2014). Identités en interaction. Limoges : Lambert-Lucas. Greenberg, M. E. (2009). A comprehensive model of the process of telephone nursing. Journal of Advanced Nursing, 65(12), 2621-2629.

Grosjean, M., \& Lacoste, M. (1999). Communication et intelligence collective. Le travail à l'hôpital. Paris: PUF.

Haakana, M. (2001). Laughter as a patient's resource. Dealing with delicate aspects of medical interaction. Text, 21(1-2), 187-219.

Haakana, M. (2010). Laughter and smiling. Notes on co-occurrences. Journal of Pragmatics, 42(6), 1499-1512.

Hall, J. K., \& Pekarek Doehler, S. (2011). Introduction. Interactional competence and development. In J. K. Hall, J. Hellermann, \& S. Pekarek Doehler (Eds.). L2 Interactional Competence and Development (pp. 206-243). Clevedon: Multilingual Matters.

Hansen, T. R., \& Bardram, J. E. (2007). Applying mobile and pervasive computer technology to enhance coordination of work in a surgical ward. Studies in Health, Technology and Informatics, 129 (1), 107-111.

Hewitt, H., McCloughan, L., \& McKinstry, B. (2009). Front desk talk. Discourse analysis of receptionist-patient interaction. British Journal of General Practice, 59, 571-577.

Holmes, J., \& Stubbe, M. (2003). Power and Politeness in the Workplace. A Sociolinguistic Analysis of Talk at Work. Essex: Pearson.

Houtkoop-Steenstra, H. (1997). Being friendly in survey interviews. Journal of Pragmatics, 28(5), 591-623.

Jefferson, G. (2004). Glossary of transcript symbols with an introduction. In G. H. Lerner (Ed.). Conversation Analysis. Studies from the First Generation (pp. 13-31). Amsterdam: John Benjamins.

Jefferson, G., Sacks, H., \& Schegloff, E. (1987). Notes on laughter in the pursuit of intimacy. In G. Button, \& J. R. E. Lee (Eds.). Talk and Social Organisation (pp. 152-205). Clevedon: Multilingual matters.

Kasper, G. (2009). When once is not enough. Politeness of multiple requests in oral proficiency interviews. Multilingua, 25, 323-350.

Lavin, D., \& Maynard, D. W. (2001). Standardization versus rapport. Respondent laughter and interviewer reaction during telephone surveys. American Sociological Review, 66(3), 453-479.

Lee, S.-H. (2009). Extended requesting. Interaction and collaboration in the production and specification of requests. Journal of Pragmatics, 41(6), 1248-1271.

Lerner, G. H. (1996). Finding "face" in the preference structures of talk-in-interaction. Social Psychology Quarterly, 59(4), 303-321.

Lindström, A. (2005). Language as social action. A study of how senior citizens request assistance with practical tasks in the Swedish home help service. In A. Hakulinen, \& M. Selting (Eds.). Syntax and Lexis in Conversation. Studies on the Use of Linguistic Resources in Talk-in-Interaction (pp. 209-230). Amsterdam : John Benjamins. 
Loriol, M. (2013). L'humour au travail : dépasser les lectures fonctionnalistes et critiques. Introduction. Les mondes du travail, 13, 13-26.

Nguyen, H. T. (2007). Rapport building in language instruction. A microanalysis of the multiple resources in teacher talk. Language and Education, 21(4), 284-303.

O'Shea, M., \& Kelly, B. (2007). The lived experiences of newly qualified nurses on clinical placement during the first six months following registration in the Republic of Ireland. Journal of Clinical Nursing, 16(8), 1534-1542.

Osvaldsson, K. (2004). On laughter and disagreement in multiparty assessment talk. Text, 24(4), 517-545.

Petitjean, C., \& González-Martínez E. (2015). Laughing and smiling to manage trouble in Frenchlanguage classroom interaction. Classroom Discourse, 6(2), 89-106.

Petitjean, C., Sterie, A., Vaucher, C., \& González-Martínez, E. (2015). « Je me permets de te déranger pour te demander un petit conseil ». La gestion de l'expertise lors d'un appel téléphonique infirmier au service de Soins intensifs. Cahiers de l'Institut de linguistique et des sciences du langage, 42, 33-56.

Pilnick, A., Hindmarsh J., \& Gill, V. (2009). « Beyond doctor and patient ». Developments in the study of healthcare interactions. Sociology of Health \& Illness, 31(6), 787-802.

Pouchelle, M.-C. (1998). « Ici on ne fait pas de cadeau ». Partages du temps et don de soi à l'hôpital. Ethnologie française, 28(4), 540-550.

Pudlinski, C. (2005). Doing empathy and sympathy. Caring responses to troubles tellings on a peer support line. Discourse Studies, 7(3), 267-288.

Raveyre, M., \& Ughetto, P. (2006). « On est toujours dans l'urgence ». Surcroît ou défaut d'organisation dans le sentiment d'intensification du travail ? In P. Askénazy, D. Cartron, F. de Coninck, \& M. Gollac (Eds.). Organisation et intensité du travail (pp. 121-128). Toulouse : Octarès. Ruusuvuori, J. (2007). Managing affect. Integration of empathy and problem-solving in health care encounters. Discourse Studies, 9(5), 597-622.

Sacks, H. (1966). The Search for Help. No-One to Turn to. Unpublished PhD dissertation, Department of Sociology: University of California at Berkeley.

Sacks, H. (1987). On the preferences for agreement and contiguity in sequences in conversation. In G. Button, \& J. R. E. Lee (Eds.), Talk and Social Organisation (pp. 54-69). Clevedon: Multilingual matters.

Sacks, H. (1992). Lectures on Conversation (I-II). Oxford: Basil Blackwell.

Sacks, H., Schegloff, E. A., \& Jefferson, G. (1974). A simplest systematics for the organization of turn-taking for conversation. Language, 50(4), 696-735.

Schegloff, E. A. (1986). The routine as achievement. Human Studies, 9(2-3), 111-151.

Schegloff, E. A. (1988). Goffman and the analysis of conversation. In P. Drew, \& A. Wooton (Eds.), Erving Goffman. Exploring the Interaction Order (pp. 88-135). London: Polity Press.

Schegloff, E. A. (1992). On talk and its institutional occasions. In P. Drew, \& J. Heritage (Eds.), Talk at Work. Interaction in Institutional Settings (pp. 101-134). Cambridge: Cambridge University Press.

Schegloff, E. A. (2007). Sequence Organization in Interaction. A Primer in Conversation Analysis. Cambridge: Cambridge University Press. 
Simmel, G. (1981[1917]). La sociabilité. Exemple de sociologie pure ou formale. In Sociologie et épistémologie (pp. 121-136). Paris : PUF.

Sterie, A. C. (2015). Recalling the doctor to action. Two requesting formats employed by a nurse for making relevant the doctor's intervention. Novitas-ROYAL, 9(2), 118-137.

Stivers, T. (2008). Stance, alignment and affiliation during storytelling. When nodding is a token of affiliation. Research on Language and Social Interaction, 41(1), 31-57.

Strauss, A. L. (2001[1966]). Structure and ideology of the nursing profession. In A. L. Strauss, Professions, Work and Careers (pp. 24-67). London: Transaction Publishers.

Strauss, A. L., Fagerhaugh, S., Suczek, B., \& Wiener, C. L. (1985). Social Organization of Medical Work. Chicago: University of Chicago Press.

Ticca, A. C. (2013). Laughter in bilingual medical interactions. Displaying resistance to doctor's talk in a Mexican village. In P. Glenn, \& E. Holt (Eds.), Studies of Laughter in Interaction (pp. 107-129). London : Bloomsbury.

Vaucher, C., \& González-Martínez, E. (2015). Le téléphone. Un outil du travail infirmier à l'hôpital. Perspective soignante, (52), 41-53.

Wahlberg, A. C. (2004). Telephone Advice Nursing. Callers' Perceptions, Nurses' Experience of Problems and Basis for Assessments. Stockholm: Karolinska Institutet.

Wakefield, D. S., Wakefield, B. J., Despins, L., Brandt, J., Davis, W., Clements, K., \& Steinmann, W. (2012). A review of verbal order policies in acute care hospitals. The Joint Commission Journal on Quality and Patient Safety, 38(1), 24-33.

Wangensteen, S., Johansson, I. S., \& Nordström, G. (2008). The first year as a graduate nurse. An experience of growth and development. Journal of Clinical Nursing, 17(14), 1877-1885.

Whalen, J., Zimmerman, D., \& Whalen, M. R. (1988). When words fail. A single case analysis. Social Problems, 35(4), 335-362.

Woloshynowych, M., Davis, R., Brown, R., \& Vincent, C. (2007). Communication patterns in a UK emergency department. Annals of Emergency Medicine, 50(4), 407-413.

Zimmerman, D. H. (1998). Identity, context and interaction. In C. Antaki, \& S. Widdicombe (Eds.). Identities in Talk (pp. 87-106). London : Sage.

Zolesio, E. (2013). L'humour entre professionnels à l'hôpital. Distinction, domination et gestion de la situation. Les mondes du travail, (13), 43-56.

\section{ANNEXES}




\section{Conventions de transcription}

\begin{tabular}{|c|c|}
\hline [ & $\begin{array}{l}\text { début du chevauchement } \\
\text { fin du chevauchement } \\
\text { enchaînement }\end{array}$ \\
\hline$(0.2)$ & $\begin{array}{l}\text { silence (absence de parole), durée indiquée en secondes et dixièmes de } \\
\text { seconde }\end{array}$ \\
\hline & intonation descendante \\
\hline$?$ & intonation montante \\
\hline & intonation progressive \\
\hline & allongement \\
\hline abso- & auto-interruption \\
\hline mais & appui \\
\hline "ouais" & segment énoncé très doucement \\
\hline "ouais & commence très doucement \\
\hline Tbonjour & le ton de la voix augmente \\
\hline ฟalors & le ton de la voix descend \\
\hline$>$ mais $<$ & segment énoncé plus rapidement \\
\hline $\begin{array}{l}\text { <refusé> } \\
\text { <pourquoi }\end{array}$ & $\begin{array}{l}\text { segment énoncé plus lentement } \\
\text { commence rapidement }\end{array}$ \\
\hline h & expiration \\
\hline h & inspiration \\
\hline () & non transcrit parce qu'incompréhensible \\
\hline (maison) & transcrit de façon incomplète ou peu sûre \\
\hline ((lit)) & commentaire du transcripteur ou segment modifié par le transcripteur \\
\hline $\mathrm{e}(\mathrm{h}) \mathrm{xcuse}$ & particule de rire intégrée dans le segment \\
\hline £ciaof & egment énoncé avec une voix souriante \\
\hline
\end{tabular}

\section{NOTES}

1. "Nouvelle au poste: pratiques de mise en pertinence et d'appréciation des compétences professionnelles des jeunes infirmières lors de leurs conversations téléphoniques à l'hôpital ». Recherche faisant partie du projet Sinergia IC-You (subside FNS n 136291), réalisée à l'Université de Fribourg en partenariat avec la Haute Ecole Arc santé et l'Hôpital Neuchâtelois (Suisse). Nous remercions Marie Débois de nous avoir aidées à constituer la collection d'extraits mobilisée dans cet article et Jocelyne Berclaz de la relecture attentive d'une première version de ce texte.

2. De manière générale, ce texte retient la forme masculine pour désigner autant des individus hommes que femmes. Toutefois, pour faire référence aux membres de certaines catégories professionnelles - infirmières, pharmaciennes, assistantes sociales, transporteurs, etc. -, nous utilisons la forme masculine ou la forme féminine en fonction du sexe auquel appartient la majorité des membres des groupes en question dans l'hôpital où se déroule notre recherche.

3. La littérature conversationnelle francophone utilisant indistinctement les termes "requête " ou « demande » pour faire référence à l'activité sur laquelle portent les études citées, nous avons retenu le dernier terme qui nous paraissait mieux convenir à une activité réalisée à l'oral, très rapidement, sans suivre de procédures administratives particulières.

4. En utilisant les termes d'alignement et d'affiliation, la littérature conversationnelle sur le rire fait régulièrement référence à une grande diversité de fonctions pratiques et relationnelles. Ces deux termes ont reçu des définitions très strictes de la part de Stivers (2008). Le premier est à utiliser lorsqu'un locuteur B poursuit l'activité conversationnelle introduite par un locuteur A. Le deuxième, lorsqu'un locuteur B soutient le point de vue exprimé par un locuteur A. Dans les deux cas, il s'agit d'actions que le récepteur d'un premier tour réalise dans son tour de réponse. Dans la majorité de nos extraits, le rire intervient par contre dans des tours premiers qui introduisent une activité. Lorsqu'il intervient dans un tour de réponse, le rire ne répond pas à une activité consistant à rire ou à faire de l'humour envers laquelle le locuteur serait en train de s'aligner en se servant justement du rire. Par ailleurs, lorsque le rire intervient dans cette position, notre argument est qu'il véhicule une position différente, plus cordiale, que celle éventuellement 
manifestée par le locuteur précédant, qui lui ne rit pas. Pour ces raisons, nous avons renoncé à utiliser la problématique de l'alignement/affiliation comme cadre général d'analyse de notre collection d'extraits, tout en reconnaissant qu'elle peut-être pertinente pour quelques passages en particulier.

5. L'analyse conversationnelle a traditionnellement gardé ses distances par rapport à des problématiques en termes de "face », avançant que la notion n'était pas véritablement traitée de manière interactionnelle par Goffman (1974 [1967]), ne serait-ce que par l'absence d'analyses d'enregistrements de conversations authentiques, ce qui est d'autant plus vrai dans le cadre de la théorie de la politesse de Brown et Levinson (1987) (Schegloff, 1988). Au sein de cette approche, des chercheurs ont toutefois lancé diverses tentatives pour donner un traitement conversationnel à la notion de "face " (Lerner, 1996) ainsi qu'à celle, plus générale mais apparentée, d'identité (Zimmerman, 1998). Ces travaux sont pertinents du moment que l'on distingue les modalités relationnelles selon qu'elles engagent, dans une plus ou moins grande mesure, l'individualité des participants. Ils ne dictent toutefois pas la problématique de cet article qui se concentre, conformément aux orientations des interlocuteurs, sur l'activité de demande téléphonique et la minimisation par le rire de ses aspects problématiques, par rapport à laquelle des questions identitaires et relationnelles sont subordonnées.

6. Nous avons remplacé par des indications fictives des éléments confidentiels apparaissant dans les données, dont les noms et les prénoms des interlocuteurs des appels, de leurs unités hospitalières ou de leurs patients.

7. Le lecteur trouvera en annexe une liste de nos conventions de transcription adaptées de Jefferson (2004). Afin de rendre particulièrement visible l'objet de cet article, nous avons indiqué avec une flèche la ligne de la transcription où se situe le rire et marqué en gras soit le rire isolée soit le mot dans lequel il s'insère. Les segments énoncés avec une voix souriante sont délimités par le signe $€$.

8. Pouchelle (1998) met en avant la dimension relationnelle de la gestion du temps à l'hôpital, qu'il s'agisse de rapports soignant-patient ou entre collègues. Raveyre et Ughetto (2006) explorent le lien entre sentiment d'urgence, valeurs professionnelles et formes d'organisation du travail. Les extraits analysés dans cet article font à plusieurs reprises écho à ces travaux, la demande apparaissant comme problématique parce que risquant de perturber le travail de son récepteur, au niveau de son organisation temporelle.

9. Dans nos cinq extraits, le jeune infirmier reconnaît le caractère problématique de la demande et donc le fait qu'elle pourrait déranger l'interlocuteur. Le rire est toutefois léger, jovial et ne correspond pas au rire gêné (Adelswärd, 1989) ou nerveux (Glenn, 2013) d'une personne mal à l'aise lorsqu'elle est prise " en faute ». Même dans l'extrait 4 ci-dessous où un reproche pourrait s'insinuer, le rire semble principalement orienté vers la démonstration d'une forme d'aisance dans la réalisation de l'activité. Ceci évite de rendre apparents des actes menaçant la face des interlocuteurs et réduit du coup la pertinence de ce type d'analyse.

10. Voir l'extrait 5 ci-dessous pour un effet similaire et Haakana (2010) pour une analyse de l'interrelation entre le rire et la voix souriante.

11. Cet appel précède celui qui est reproduit dans l'extrait 3 où Tim revient sur cette demande d'annulation. 


\section{RÉSUMÉS}

Cet article se base sur l'analyse conversationnelle d'un corpus d'appels téléphoniques entre de jeunes infirmières diplômées, qui se trouvent dans la première année d'engagement à l'hôpital, et d'autres membres du personnel hospitalier. L'activité principale de ces appels est une demande de l'infirmière adressée à son interlocuteur. Lors de ces appels, il arrive fréquemment que l'infirmière produise des particules de rire - avant, pendant ou après un tour de parole - qui ne sont pas associées à des activités humoristiques telles que des blagues ou des moqueries, et qui ne sont ni précédées ni suivies de rire par l'interlocuteur de l'infirmière. Ces rires, brefs et ténus, sont parfois associés à la production par l'infirmière de tours de parole avec une voix souriante et/ou à des ressources langagières telles que des formules de politesse. Dans cet article, nous examinons ces rires dans plusieurs contextes séquentiels en mettant en évidence leur rapport avec l'activité de demande accomplie lors de l'appel. Ces rires permettent à l'infirmière de minimiser des aspects problématiques de la demande ou de son traitement, en introduisant une touche de cordialité dans ses interventions. Les dimensions pratiques et relationnelles du rire concourent à l'avancement de l'activité, ce vers quoi s'oriente l'interlocuteur de l'infirmière dans sa réponse. Cet article contribue ainsi à la réflexion sur les fonctions du rire en contexte professionnel ainsi qu'à l'étude des compétences interactionnelles mobilisées par la jeune infirmière dans ses activités professionnelles.

This article is based on the conversation analysis of a corpus of telephone calls between young nursing graduates in their first year of work at a hospital and other members of the hospital staff. The main activity of these calls is a request that the nurse makes of her interlocutor. During these calls, the nurse often produces laughter particles - before, during and after a turn-at-talk that are not associated with humorous events such as jokes or teasing and are not preceded or followed by laughter from the nurse's interlocutor. This laughter, brief and light, is sometimes associated with the production, by the nurse, of smiling-voice turns and/or linguistic resources such as polite formulae. In this article, we examine this laughter in several sequential contexts, demonstrating its relationship to the request activity accomplished through the call. This laughter allows the nurse to minimize the problematic aspects of the request or its processing by adding a touch of cordiality. The practical and relational aspects of laughter support the progress of the activity, towards which the nurse's interlocutor is oriented during his or her response. This article thus contributes to reflection on the functions of laughter in professional settings, as well as to the study of the interactional competences deployed by young nurses in their professional activities.

\section{INDEX}

Keywords : laughter, cordiality, phone request, young nurse, conversation analysis

Mots-clés : rire, cordialité, demande téléphonique, jeune infirmière, analyse conversationnelle 


\section{AUTEURS}

\section{ESTHER GONZÁLEZ-MARTÍNEZ}

Département des sciences sociales - Université de Fribourg, route des Bonnesfontaines 11

CH-1700 Fribourg - esther.gonzalezmartinez@unifr.ch

\section{CÉCILE PETITJEAN}

Centre de linguistique appliquée - Université de Neuchâtel, rue Pierre-à-Mazel 7 CH-2000 Neuchâtel - cecile.petitjean@unine.ch 